EPJ Web of Conferences 59, 13014 (2013)

DOI: $10.1051 /$ epjconf/20135913014

(C) Owned by the authors, published by EDP Sciences, 2013

\title{
On the absolute calibration of a DT fusion neutron yield diagnostic
}

\author{
C.L. Ruiz ${ }^{1}$, R.J. Leeper ${ }^{1}$, G.W. Cooper ${ }^{2}$, G.A. Chandler ${ }^{1}$, C.M. Cox ${ }^{1}$, \\ B.L. Doyle ${ }^{1}$, D.L. Fehl ${ }^{1}$, K.D. Hahn ${ }^{1}$, B.R. McWatters ${ }^{1}$, A.J. Nelson ${ }^{2}$, \\ R.M. Smelser ${ }^{1}$, C.S. Snow ${ }^{1}$, J.A. Torres ${ }^{1}$, D.L. Bleuel ${ }^{3}$ and J.D. Kilkenney ${ }^{4}$ \\ ${ }^{1}$ Sandia National Laboratories, Albuquerque, New Mexico, USA \\ 2 University of New Mexico, Albuquerque, New Mexico, USA \\ ${ }^{3}$ Lawrence Livermore National Laboratories, Livermore, California, USA \\ ${ }^{4}$ General Atomics, San Diego, California, USA
}

\begin{abstract}
Recent advances in Inertial Confinement Fusion (ICF) experiments at Lawrence Livermore National Laboratory's National Ignition Facility (NIF) have underscored the need for accurate total yield measurements of DT neutrons because yield measurements provide a measure of the predicted performance of the experiments. Future gas-puff DT experiments at Sandia National Laboratory's Z facility will also require similar measurements. For ICF DT experiments, the standard technique for measuring the neutron $(14.1 \mathrm{MeV})$ yield, counts the activity (counts/minute) induced in irradiated copper samples. This activity occurs by the ${ }^{63} \mathrm{Cu}(\mathrm{n}, 2 \mathrm{n}){ }^{62} \mathrm{Cu}$ reaction where ${ }^{62} \mathrm{Cu}$ decays by positrons $\left(\beta^{+}\right)$with a half-life of 9.67 minutes. The calibrations discussed here employ the associated-particle method (APM), where the $\alpha\left({ }^{4} \mathrm{He}\right)$ particles from the $\mathrm{T}(\mathrm{d}, \mathrm{n})^{4} \mathrm{He}$ reaction are measured to infer neutron fluxes on a copper sample. The flux induces ${ }^{62} \mathrm{Cu}$ activity, measured in a coincidence counting system. The method leads to a relationship between a DT neutron yield and copper activity known as the $F$-factor. The goal in future experiments is to apply this calibration to measure the yield at NIF with a combined uncertainty approaching $5 \%$.
\end{abstract}

\section{Introduction}

We have developed a Lawrence Livermore's National Ignition Facility (NIF) DT neutron diagnostic (NAD20), based on the activation of copper via ${ }^{63} \mathrm{Cu}(\mathrm{n}, 2 \mathrm{n}){ }^{62} \mathrm{Cu}$ reaction with a half-life of $9.67 \mathrm{~min}$. for ${ }^{62} \mathrm{Cu}$ and a dedicated nuclear electronics counting system. We report here progress on a technique to calibrate the activation of copper and the counting system. A description of the counting system is described elsewhere [1-3]. The calibration technique employing the associated-particle method (APM) and the $F$-factor method follow in Sections 1 and 2 below with recent results and concluding remarks given in Section 3.

\section{ASSOCIATED-PARTICLE METHOD (APM)}

The $\mathrm{T}(\mathrm{d}, \mathrm{n})^{4} \mathrm{He}$ fusion reaction has been employed as a source of $14.1 \mathrm{MeV}$ neutrons for the $\mathrm{Cu}$ calibrations. Deuterium $\left(\mathrm{d}^{+}\right)$ions were provided by a $300 \mathrm{keV}$ Cockcroft-Walton accelerator, located at Sandia National Lab's Ion Beam Laboratory and delivered to a tritiated target. The $\mathrm{d}^{+}$ions at $175 \mathrm{keV}$, magnetically analyzed, were electrostatically focused onto a 1-cm diam., 2.6- $\mu \mathrm{m}$ thick, tritium loaded erbium (Er) target, layered over a 0.75 inch diameter oxygen-free 50 mil thick copper disk. The targets, centered in the $\mathrm{d}^{+}$beam, were in contact with a water-cooled copper cold-finger. A precision-built,

This is an Open Access article distributed under the terms of the Creative Commons Attribution License 2.0, which permits unrestricted use, distribution, and reproduction in any medium, provided the original work is properly cited. 


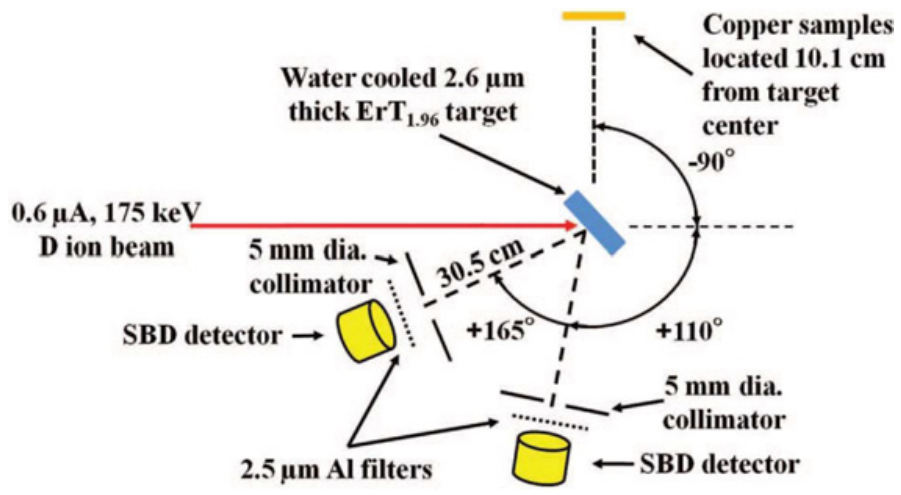

Figure 1. Experimental setup.

thin-walled vacuum chamber was employed for the experiments. A residual-gas-analyzer (RGA) was used to monitor tritium de-sorption due to target beam heating. To infer neutron yield from the $\mathrm{T}(\mathrm{d}, \mathrm{n})^{4} \mathrm{He}$ reaction, the APM required a measurement of the corresponding $\alpha$ counts in the energy spectrum. The $\alpha$ energy spectrum obtained in these calibrations was measured by placing two co-planar chargeparticle detectors at $110^{\circ}$ and $165^{\circ}$ relative to the $0^{\circ}$ beam direction. Ion-implanted surface barrier detectors (SBD, 100- $\mu \mathrm{m}$ thick with $50-\mathrm{mm}^{2}$ active areas) were employed. Aluminum filters $(2.6-\mu \mathrm{m}$ thick) were placed in front of the SBDs to prevent detection of Rutherford scattered $\mathrm{d}^{+}$ions from the target. The SBD's were located $30.48 \mathrm{~cm}$ from the target center. The normal of each SBD's axis intercepted the front-facing surface of the target center regardless of the target's orientation relative to the detector's front surface, and both detectors viewed the target at equal geometric solid angles. Protective baffles prevented $\alpha$ scattering into the SBDs. Solid angles were pre-determined by measuring the SBDsubtended activity produced by a calibrated (3\%) ${ }^{241} \mathrm{Am} \alpha$-emitting source yielding an average value between the two detectors of $(2.258 \pm 0.068) \times 10^{-4} \mathrm{sr}$, where the noted uncertainty is due to the source. Copper samples (1-mm thick by $5 \mathrm{~cm}$ diameter), placed in light-weight holders, were located $10.1 \mathrm{~cm}$ from the target center and at $90^{\circ}$ relative to the $\mathrm{d}^{+}$beam. Including the target holder, the chamber wall, and the $\mathrm{Cu}$ sample holder, MCNP [4] calculations indicated a combined 3\% reduction of the neutron flux on the $\mathrm{Cu}$ samples due to neutron scattering and attenuation. The experimental schematic is shown in Figure 1.

The APM employed both theoretical and experimental components. The absolute calculations are described as follows. Based on the target thickness and density, and the $\mathrm{T}(\mathrm{d}, \mathrm{n})^{4} \mathrm{He}$ nuclear reaction, both the $\alpha$ particle $(\alpha / \mu \mathrm{C})$ per msr or neutron yield (neutrons/ $\mu \mathrm{C}$ ) per msr at a selected laboratory angle are

$$
\frac{d Y}{d \Omega}=\int_{E_{d}^{\max }}^{0} \nu \frac{d \sigma(E) / d \Omega}{d E / d x} d E
$$

where $v$ is the stoichiometric target loading ratio (i.e., the number of deuterons per erbium atom), $d \sigma(E) / d \Omega$ is the laboratory differential cross-section [5] for the $\mathrm{T}(\mathrm{d}, \mathrm{n})^{4} \mathrm{He}$ reaction at $\mathrm{d}^{+}$kinetic energy $E, E_{d}^{\max }$ is the initial energy of the $\mathrm{d}^{+}$, and $d E / d x$ is the stopping power of the tritium loaded erbium target. The loading ratio $v$ for these targets has been determined to be 1.96 [6]. The stopping power used was a modified Bragg's-rule expression for ErT ${ }_{1.96}$ and described elsewhere [7]. Differential crosssections, kinematics, and solid angles of the reaction were done in the center-of-mass system and then transformed to the laboratory system to determine the $\alpha$ and neutron emission-angle yield. Equation (1) was evaluated numerically. The calculated yields for $\alpha$ particles $\left(Y_{\alpha}^{\text {calc }}\right)$ at $110^{\circ}$ and $165^{\circ}$ and for neutrons $\left(Y_{n}^{\text {calc }}\right)$ at 90 degrees from the $E_{d}^{\max }=175 \mathrm{keV} \mathrm{d}+$ kinetic energies were 11180 and $9690(\alpha / \mu \mathrm{C})$ per msr and 11820 (neutrons/ $\mu \mathrm{C}$ ) per msr respectively. These values were then used to determine the 
experimental neutron yield $Y_{n}$ incident on a copper sample:

$$
Y_{n}=\left(C_{\alpha}-B_{\alpha}\right) \cdot\left(Y_{n}^{\text {calc }} / Y_{\alpha}^{\text {calc }}\right) \cdot\left(\Omega_{n} / \Omega_{\alpha}\right)
$$

where $C_{\alpha}$ are $\alpha$ particle counts detected, $B_{\alpha}$ are background counts, and $\Omega_{n}$ and $\Omega_{\alpha}$ are the solid angles subtended by the copper samples and $\alpha$ detectors, respectively.

Standard nuclear electronics were used to monitor the target current, the SBD detector $\alpha$ count rate and the $\alpha$ energy spectra. The SBD count rates were obtained by multi-channel scaling. The $\alpha$ particle energy spectra were collected for both detectors in separate multi-channel-analyzers. Currents were monitored with a current integrator where the targets were biased with +300 volts and surrounded by a -60 volt biased screen. These biases suppressed secondary-electron production by the $\mathrm{d}^{+}$beam near and at the target, thus preventing incorrect target current readings. Although the calibration method here does not require a beam current value, it was necessary to compare the measured current charge rate on target (C/s) with the SBD's $\alpha$ count rates $(\alpha / \mathrm{s})$ so that changes in $\alpha$ rates with no drop in C/s would identify changes in the target-tritium loading ratio. This procedure also measured the temporal drift of the inferred neutron flux (neutrons/s) per $\mathrm{cm}^{2}$ on the irradiated copper sample.

\section{F-FACTOR METHOD}

The $\mathrm{Cu}$ activation process may be formally described by

$$
\left(C_{\text {coin }}-B_{\text {coin }}\right)=\phi \varepsilon_{A} \varepsilon_{D} \varepsilon_{S} \varepsilon_{B} M N_{A} \sigma(E)\left[\left(1-e^{-\lambda t_{0}}\right)\left(e^{-\lambda t_{1}}-e^{-\lambda t_{2}}\right)\right] / \lambda A_{W} .
$$

This equation indirectly describes the copper positron decays, which are counted by annihilation into two coincident $511 \mathrm{keV}$ gamma rays in a coincidence-counting system [1,2]. The fundamental parameters in Eq. (3) for the ${ }^{63} \mathrm{Cu}(\mathrm{n}, 2 \mathrm{n}){ }^{62} \mathrm{Cu}$ reaction are as follows: (a) the neutron flux is $\varphi$; (b) the quantities $\varepsilon_{A}, \varepsilon_{D}, \varepsilon_{S}, \varepsilon_{B}$, are the isotopic abundance of ${ }^{63} \mathrm{Cu}$ in natural copper, the detector efficiency of the coincidence counting system, the self-attenuation of positrons and $511 \mathrm{keV}$ gamma-rays in the copper volumetric sample, and the positron branching ratio for ${ }^{62} \mathrm{Cu}$ nuclei, respectively [3]; (c) the mass (grams) and atomic weight of copper are given by $M$ and $A_{W}$; (d) $N_{A}$ is Avogadro's number; (e) the $\mathrm{Cu}$ neutron irradiation time is $t_{0}$; (f) $t_{1}$ and $t_{2}$ define the start and stop time after $t_{0}$ used for determining the coincidence-counting interval of $C_{\text {coin }}-B_{\text {coin }} ;(\mathrm{g})$ the cross-section is given by $\sigma(E) ;(\mathrm{H})$ and the ${ }^{62} \mathrm{Cu}$ half-life $\left(\tau_{1 / 2}=9.67 \mathrm{~min}\right.$. $)$ is defined in $\lambda=.693 / \tau_{1 / 2}$ The coincidence counting $\left(C_{\text {coin }}-B_{\text {coin }}\right)$ in Eq. (3) may also be re-written as

$$
F=\frac{\left(C_{\text {coin }}-B_{\text {coin }}\right)}{\varphi M\left(1-e^{-\lambda t_{0}}\right)\left(e^{-\lambda t_{1}}-e^{-\lambda t_{2}}\right)} \lambda
$$

where the neutron flux $\varphi$ is determined from $Y_{n} /\left[t_{0} \times \mathrm{Cu}\right.$ sample area $\left.\left(\mathrm{cm}^{2}\right)\right]$. Eq. (4) may alternatively be written as

$$
F=\varepsilon_{A} \varepsilon_{D} \varepsilon_{S} \varepsilon_{B} \sigma(E) N_{A} / A_{W}
$$

which is called the $F$-factor. The units are in (counts/neutron) $\left(\mathrm{cm}^{2} / \mathrm{g}\right)$. Special features of Eq. (5) are (a) it contains the reaction cross-section for copper activation; (b) it also contains the coincidencedetector efficiency and the copper sample self-absorption factor. But, the $F$-factor, instead, is determined exclusively by measured and known quantities in Eq. (4) and defines the calibration (coincidence counts to neutron yield) of the activated copper counting system.

\section{EXPERIMENTAL RESULTS AND CONCLUSIONS}

Four preliminary experiments were recently conducted to determine an $F$-factor. Copper samples (of the NIF NAD20 diagnostic design, $1.0-\mathrm{mm}$ thick by $5.0-\mathrm{cm}$ diameter and $M=18.4 \mathrm{~g}$ ) were irradiated with DT neutrons from $t_{0}=600$ to 1200 seconds. Target currents and $\alpha$ particle detection rates at 110 
and 165 degrees were monitored for drift. A typical target current was $600 \mathrm{nC} / \mathrm{s}$ with a $\pm 0.5 \%$ fluctuation indicating excellent temporal stability and implying a steady neutron production and excluding temporal corrections to Eq. (4). After irradiation, the copper samples were retrieved within a few minutes and placed in the coincidence counting system. The measured quantities $\left(C_{\text {coin }}-B_{\text {coin }}\right), t_{0}, t_{1}, t_{2}$, etc. in Eq. (4), were then used to determine $F$-factors. The results for the $110^{\circ}$ and $165^{\circ}$ degree detectors from repeated irradiations, gave an average value of $3.86 \times 10^{-4}$ with standard deviation of $0.11(2.9 \%)$ indicating good repeatability. The presently identified systematic uncertainties are estimated at (a) $3 \%$ for $\Omega_{\alpha}$, (b) $3 \%$ for $\nu$, (c) <1\% for counting statistics, (d) $2 \%$ for $\Omega_{n}$, (e) and 3\% for $Y_{n}$ [6]. Combined in quadrature, the preliminary $F$-factor uncertainty is thus $\sim 6 \%$.

In conclusion, the $F$-factor determined here, when applied to the NIF NAD20 diagnostic, may meet the design goal of a 5\% combined uncertainty. The calibration method presented here is capable of measuring NIF DT neutron yields as low as $10^{13}$ with copper samples at $19 \mathrm{~m}$ from the source, and in the present NIF configuration extends to $\sim 10^{16}$. We, however, acknowledge that transferring the $F$-factor of the activated $\mathrm{Cu}$ counting-system presented here to NIF experiments will require additional neutron attenuation and scattering corrections, and that larger source-to-copper sample distances with longer recovery times $t_{1}$ may yield lower counts and thus impact the measured yield uncertainty. The experimental application of the $F$-factor method and a description of the transfer of the calibration to NIF experiments to determine neutron yields are reported elsewhere [3].

Sandia National Laboratories is a multi-program laboratory managed and operated by Sandia Corporation, a wholly owned subsidiary of Lockheed Martin Corporation, for the U.S. Department of Energy's National Nuclear Security Administration under contract DE-AC04-94AL85000.

\section{References}

[1] C. L. Ruiz, et al., Rev. Sci. Instrum. 63, 4889 (1992)

[2] G. W. Cooper and C. L. Ruiz, Rev. Sci. Instrum. 72, No. 1, 814 (2001)

[3] R. J. Leeper, to be published in these proceedings

[4] J. F. Briesmeister, MCNP- A General Monte Carlo Neutron-Particle Transport Code, Version 4C, Los Alamos National Laboratory, 2000

[5] H. Liskien and A. Paulsen, Nuclear Data Tables 11, No. 7, 569 (1973)

[6] D. Malbrough, private communication

[7] D. Malbrough, et al., Nucl. Instr. Meth. B28, 459 (1987) 